\title{
Efectos a un año de un programa educativo en el control metabólico de pacientes con diabetes de un servicio especializado en un hospital nacional peruano
}

Effects at one year of an educational program on the metabolic control of patients with diabetes in a specialized service of a Peruvian national hospital

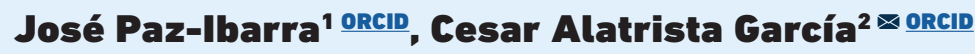

Fecha correspondencia:

Recibido: octubre 14 de 2020. Revisado: diciembre 12 de 2020. Aceptado: enero 20 de 2021.

Forma de citar:

Paz-Ibarra J, Alatrista García C. Efectos a un año de un programa educativo en el control metabólico de pacientes con diabetes de un servicio especializado de un hospital nacional peruano. Rev CES Med. 2021; 35(1): 4-15.

$\underline{\text { Open access }}$

(C) Derecho de autor

Licencia creative commons

Ética de publicaciones

Revisión por pares

Gestión por Open Journal System

DOl: http://dx.doi.org/10.21615/

cesmedicina.35.1.1

ISSN 0120-8705

e-ISSN 2215-9177

\section{Resumen}

Objetivo: determinar los efectos en el control de la diabetes durante un año de seguimiento de un grupo de pacientes que recibieron un programa educativo añadido al tratamiento habitual en la consulta ambulatoria especializada del Hospital Nacional "Edgardo Rebagliati Martins" en Perú. Metodología: estudio cuasiexperimental en el que se distribuyeron 90 pacientes en dos grupos: intervención o control. El primero recibió cuatro sesiones educativas en grupos de cinco a ocho participantes. Se procuró que la terapia farmacológica se mantuviera sin modificaciones significativas tres meses antes y durante todo el seguimiento. Se determinaron los cambios en los parámetros clínico-bioquímicos principalmente $\mathrm{HbA} 1 \mathrm{c}$ al inicio, a los seis y 12 meses después de la intervención. Resultados: todos los pacientes completaron todas las sesiones, así como las consultas de seguimiento con la respectiva toma de muestras para la determinación de los parámetros bioquímicos. En el grupo de intervención la HbA1c disminuyó $1,2 \%$ a los 12 meses, mientras el grupo control presentó un incremento de $0,5 \%$. El peso, la glicemia en ayunas y el colesterol LDL también disminuyeron durante el seguimiento; el porcentaje de pacientes que alcanzaron las metas de HbA1c aumentó en $22 \%$ y de LDL en $11 \%$ a los 12 meses de seguimiento. Conclusiones: un programa de educación diabetológica añadido al manejo convencional especializado en un hospital nacional de referencia mejoró el control metabólico durante un año de seguimiento.

Palabras clave: Educación en salud; Diabetes mellitus; Educación del paciente; Manejo de la enfermedad; Perú.

\section{Abstract}

Objective: To determine the effects on the control of diabetes during one year of follow-up of a group of patients who received an educational program added to the usual treatment in the specialized outpatient consultation of the National Hospital "Edgardo Rebagliati Martins", Peru. Methodology: 


\section{Sobre los autores:}

1. Endocrinólogo. Magíster en Docencia e Investigación en Salud. Profesor Asociado de la Universidad Nacional Mayor de San Marcos.

2. Bachiller en Medicina. Residente de Endocrinología del Hospital Nacional Edgardo Rebagliati Martins.
Son múltiples los beneficios que trae consigo capacitar a los pacientes con diabetes mellitus, así, la educación ejerce efectos favorables como cambios en los estilos de vida: alimentación saludable, realización de actividad física y abandono de hábitos nocivos.
Quasi-experimental study, 90 patients were distributed in 2 groups: intervention versus control. The intervention group received 4 educational sessions in groups of 5-8 participants. Pharmacological therapy was maintained without significant modifications 3 months before and throughout the follow-up. Changes in the clinical-biochemical parameters were determined, mainly $\mathrm{HbA} 1 \mathrm{c}$ at the beginning, at 6 and 12 months after the intervention. Results: The 90 patients completed all the sessions and follow-up consultations with the collection of samples for the determination of the biochemical parameters. In the intervention group, $\mathrm{HbA} 1 \mathrm{c}$ decreased $1.2 \%$ at 12 months while the control group showed an increase of $0.5 \%$; weight, fasting blood glucose, and LDL cholesterol also decreased during follow-up; the percentage of patients who reached the HbA1c goals increased by $22 \%$ and LDL by $11 \%$ at 12 months of follow-up. Conclusions: A diabetes education program added to the conventional specialized management in a national referral hospital improved metabolic control during one year of follow-up.

Keywords: Health Education; Diabetes mellitus; Patient education; Disease Management; Peru.

\section{Introducción}

La diabetes mellitus es un problema de salud pública. Se proyecta a más de 49 millones de personas con la enfermedad para el 2045 en Latinoamérica (1) y en el Perú se proyectan más de dos millones para 2030. Un estudio de prevalencia nacional reporta un $7 \%$ en mayores de 25 años (2). Un programa de vigilancia epidemiológica de la enfermedad muestra que en algunos hospitales existía un alto número de pacientes con un inadecuado control glicémico y una baja adherencia al tratamiento (3).

La educación diabetológica es un conjunto de actividades dirigidas a estos pacientes para promover habilidades y conductas saludables, así como para alcanzar una adecuada adherencia al tratamiento. Tiene que ser apoyada por actividades grupales estructuradas, sistemáticas y participativas que faciliten el intercambio de experiencias entre los pacientes, el apoyo mutuo y la motivación en el seguimiento de las medidas terapéuticas $(4,5)$. La tarea de educación al paciente deberá iniciarse en el momento del diagnóstico, durará toda la vida y responderá a las etapas específicas por las que atraviesa (debut, descompensación, complicaciones asociadas, embarazo, comorbilidades) (6).

Son múltiples los beneficios que trae consigo capacitar a los pacientes con diabetes mellitus, así, la educación ejerce efectos favorables como cambios en los estilos de vida: alimentación saludable, realización de actividad física y abandono de hábitos nocivos $(4,5)$.

El ensayo clínico eXpert Patient Education versus Routine Treatment (X-PERT) en 314 pacientes con diabetes mellitus tipo 2 compara la educación individual versus un programa de educación grupal (seis sesiones de dos horas/semana). A los 14 meses el programa X-PERT, comparado con la atención habitual, mostró un descenso de la hemoglobina glicosilada A1c ( $\mathrm{HbA} 1 \mathrm{C})(-0,6 \%$ vs. $+0,1 \%)$ y una pérdida de 0,5 kg de peso. También se observó una disminución en la necesidad de antidiabéticos (7).

El estudio Diabetes Education and Self Management for Ongoing and Newly Diagnosed (DESMOND) evaluó un programa educativo con énfasis en cambios en su estilo de vida y en la modificación de factores de riesgo cardiovascular. El programa consistió en un total de seis horas de educación grupal a pacientes con diagnóstico de diabetes 
Las características del programa fueron: educación grupal (5-8 pacientes por grupo) impartida en base a sesiones educativas interactivas y participativas, en forma de charla-taller de 60 minutos cada una. Las sesiones fueron desarrolladas por un solo educador certificado, promoviendo la mayor participación posible y resolviendo las dudas que pudieran surgir. mellitus tipo 2 de 12 semanas o menos. Comparado con la intervención habitual y después de un año de seguimiento se observó una reducción de HbA1c en ambos grupos con diferencia no significativa, así como reducción de peso (8).

El objetivo de este estudio fue determinar los efectos a un año de seguimiento de un programa educativo añadido al tratamiento habitual especializado en el control de pacientes con diabetes mellitus del Hospital Nacional "Edgardo Rebagliati Martins" (HNERM) en Lima- Perú.

\section{Materiales y métodos}

Estudio cuasiexperimental tipo antes/después.

Criterios de inclusión: pacientes con diabetes mellitus tipo 2, edad: 40-65 años; tiempo de diagnóstico: menos de 10 años; referidos al servicio de endocrinología con diagnóstico de diabetes no controlada ( $\mathrm{HbA1c} 7,5-12,9 \%$ ); presión arterial controlada (menor de $140 / 90 \mathrm{~mm} \mathrm{Hg}$ ) en los últimos seis meses e índice de masa corporal (IMC) menor de $35 \mathrm{~kg} / \mathrm{m}^{2}$.

Criterios de exclusión: glicemia mayor de 300 mg/dL o sintomático; HbA1c mayor de $13 \%$; que no estuvieran referidos o que tuvieran más de un año siendo atendidos en la institución; que hayan participado de programas educativos estructurados; con complicaciones crónicas incapacitantes (amaurosis, terapia de diálisis, pie diabético infectado o amputaciones), déficit sensorial, psicológico o físico que no permita participar en las sesiones.

Considerando un nivel de confianza de $95 \%$, una potencia de $80 \%$, una prevalencia de éxito esperada (proporción de pacientes que presentan una disminución de la concentración de $\mathrm{HbA} 1 \mathrm{c}$ ) de 50 \% con la intervención educativa añadida al tratamiento habitual y de $25 \%$ sólo con el tratamiento habitual, se calculó una muestra de 45 pacientes para cada grupo (9). Los sujetos de la muestra fueron asignados de forma no aleatoria según orden de llegada y factibilidad de cumplir con las sesiones educativas.

La variable independiente fue el programa de educación diabetológica (PED). La principal variable dependiente fue la $\mathrm{HbA} 1 \mathrm{c}$; las secundarias fueron cambios en el peso, IMC, glicemia en ayunas y perfil lipídico.

Intervención: El PED se basó en los siete comportamientos de autocuidado del paciente recomendados por la Asociación Americana de Educadores en Diabetes y la Asociación Americana de Diabetes: estando activo, comiendo sano, tomando los medicamentos, monitoreando resultados, resolviendo problemas, reduciendo riesgos, afrontando saludablemente su enfermedad (10-13).

Las características del programa fueron: educación grupal (5-8 pacientes por grupo) impartida en base a sesiones educativas interactivas y participativas, en forma de charla-taller de 60 minutos cada una. La distribución del tiempo fue: 2-5 minutos: bienvenida y resolución de preguntas de sesión anterior; 20-30 minutos; presentación del contenido de la sesión, exposición dialogada participativa; 10-15 minutos: charla motivacional, ejercicios de estiramiento y relajación, demostraciones prácticas; 5-10: minutos solución de preguntas, dudas y repaso de conclusiones; 3-5 minutos: programación de siguiente sesión, control de glucosa, despedida. El contenido de 
Se encontraron diferencias en la HbA1c: $-1,2 \%$ en el grupo de intervención vs. $+0,5 \%$ en el grupo control a los 12 meses. El peso, IMC, glicemia en ayunas y colesterol HDL (HDLC) a los seis meses también evidenciaron mejoría significativa. cada sesión fue así: primera sesión: qué es la diabetes mellitus, tipos de diabetes mellitus, factores de riesgo, metas de control; segunda sesión: alimentación saludable; tercera sesión: actividad física, cuidados del pie e hipoglicemia y, cuarta sesión: adherencia al tratamiento, resumen de las tres sesiones anteriores en base a lo realizado por Hernández et al. (14).

Las sesiones fueron desarrolladas por un solo educador certificado, promoviendo la mayor participación posible y resolviendo las dudas que pudieran surgir durante la sesión.

La información fue almacenada en Microsoft Excel 2010 (Microsoft Corp ${ }^{\circledR}$ ) mediante doble digitación comparativa. Todos los análisis estadísticos se realizaron en software Stata $^{\circledR}$ (Stata Corp, 2011, Stata 12 Base Reference Manual, College Station, TX, USA). Se desarrolló un análisis univariado de las variables cuantitativas y cualitativas correspondiente a cálculos de medidas de resumen y medidas de dispersión, así como frecuencias absolutas y relativas. El análisis bivariado, utilizando las pruebas de T de student, chi cuadrado, prueba exacta de Fisher o Wilcoxon para variables con distribución normal y U-Mann Withney para variables que no tuvieran distribución normal, permitió establecer una relación entre variables. Un valor $p<0,05$ fue considerado como significativo en la relación entre las variables analizadas.

El estudio fue aprobado por el Comité de Ética e Investigación del HNERM, respetándose los principios bioéticos para investigaciones en salud. Todos los pacientes leyeron y firmaron el consentimiento informado.

\section{Resultados}

Los 90 pacientes completaron el seguimiento. Las características demográficas, clínicas y bioquímicas basales de ambos se presentan en el cuadro 1, no encontrándose diferencias en todos los parámetros, excepto en la concentración sanguínea de triglicéridos que fueron mayores en el grupo de intervención $(p=0,03)$.

La comparación de los cambios registrados en los parámetros clínicos y bioquímicos entre ambos grupos se presenta en el cuadro 2, encontrando diferencias en los niveles de HbA1c y glicemia en ayunas a los 12 meses a favor del grupo de intervención.

Se encontraron diferencias en la HbA1c: - 1,2 \% en el grupo de intervención vs. +0,5\% en el grupo control a los 12 meses. El peso, IMC, glicemia en ayunas y colesterol HDL (HDLC) a los seis meses también evidenciaron mejoría significativa; mientras que al año una diferencia significativa se presentó en el colesterol total en el grupo PED-HNERM (cuadro 3). 
Cuadro 1. Características basales

La meta de $\mathrm{HbA} 1 \mathrm{c}<7 \%$ se alcanzó en el 24,4 y $22,2 \%$ en el grupo PED vs. 4,4 y $6,7 \%$ en el grupo control, a los seis y 12 meses, respectivamente.

\begin{tabular}{|c|c|c|c|}
\hline \multirow{3}{*}{$\begin{array}{l}\text { Características } \\
\text { Edad }{ }^{p 50[R \mid c]}\end{array}$} & \multicolumn{2}{|c|}{ Grupo } & \multirow{2}{*}{$\begin{array}{c}p \\
=45)\end{array}$} \\
\hline & \multirow{2}{*}{$\begin{array}{c}\text { Control }(n=45) \\
63(65-56)\end{array}$} & PED-HNERM $(n=45)$ & \\
\hline & & $64(65-58)$ & $0,42^{\mathrm{a}}$ \\
\hline Sexo ${ }^{n(\%)}$ & & & $0,81^{b}$ \\
\hline Femenino & $34(75,6)$ & $35(77,8)$ & - \\
\hline Años de diagnóstico p5o[RIC] & $10(10-10)$ & $10(10-3)$ & $0,05^{\mathrm{a}}$ \\
\hline Grado de instrucción superior n(\%) & $34(75,5)$ & $31(68,9)$ & $0,92^{b}$ \\
\hline Uso de metformina ${ }^{n(\%)}$ & $36(80)$ & $38(84,4)$ & $0,54^{b}$ \\
\hline Insulinoterapia & $33(73,3)$ & $32(71,1)$ & $0,84^{b}$ \\
\hline Uso de IECA/ARA-2 n(\%) & $34(75,6)$ & $30(66,7)$ & $0,65^{b}$ \\
\hline Uso de estatinas ${ }^{n(\%)}$ & $36(80,0)$ & $35(77,8)$ & $0,99^{b}$ \\
\hline Uso de AAS n(\%) & $45(100)$ & $45(100)$ & $1^{\mathrm{b}}$ \\
\hline Peso $(k g)^{x(D S)}$ & $70,98(11,12)$ & $71,75(12,70)$ & $0,76^{c}$ \\
\hline $\operatorname{IMC}\left(\mathrm{kg} / \mathrm{m}^{2}\right)^{x(D S)}$ & $28,20(3,49)$ & $27,74(27,74)$ & $0,56^{c}$ \\
\hline $\mathrm{HbA} 1 \mathrm{C}(\%)^{\mathrm{P} 50[\mathrm{RIC}]}$ & $9,2(10,3-8,2)$ & $9,5(10,6-8,5)$ & $0,19 a$ \\
\hline Glucosa (mg/dl) $)^{\text {550[RIc] }}$ & $168(238-126)$ & $186(201-133)$ & $0,84^{\mathrm{a}}$ \\
\hline Colesterol Total (mg/dl) $)^{\mathrm{x(DS})}$ & $189,53(52,44)$ & $183,44(40,38)$ & $0,54^{c}$ \\
\hline $\operatorname{LDLc}(\mathrm{mg} / \mathrm{dl})^{\mathrm{x}(\mathrm{DS})}$ & $113,82(43,63)$ & $108,51(34,32)$ & $0,52^{c}$ \\
\hline HDLc $(\mathrm{mg} / \mathrm{dl})^{\mathrm{P50[RIC]}}$ & $45(50-38)$ & $39(47-38)$ & $0,29^{a}$ \\
\hline Triglicéridos (mg/dl) ${ }^{\text {P5o[Rlc] }}$ & $154(193-104)$ & $180(281-134)$ & $0,03^{a}$ \\
\hline
\end{tabular}

PED: Programa de Educación Diabetológica. p50: mediana, RIC: rango intercuartílico; x: promedio, DS: desviación estándar ADOS: antidiabéticos orales; IECA: inhibidores de la enzima convertidora de angiotensina; ARA-2: antagonistas del receptor de angiotensina 2; AAS: Acido acetil salicílico; IMC: índice de masa corporal; HbA1C: Hemoglobina glicosilada A1c; LDLc: colesterol de baja densidad, HDLc: colesterol de alta densidad.

a. U Mann Whitney, b. chi cuadrado, c. T-student 
Cuadro 2. Cambios en los parámetros clínicos y bioquímicos

\begin{tabular}{|c|c|c|c|}
\hline \multirow{2}{*}{ Características } & \multicolumn{2}{|c|}{ Grupo } & \multirow{2}{*}{$p$} \\
\hline & Control $(n=45)$ & Intervención $(n=45)$ & \\
\hline \multicolumn{4}{|c|}{ A los seis meses } \\
\hline $\mathrm{HbA} 1 \mathrm{C}(\%)^{\mathrm{P} 50[\mathrm{RIC}]}$ & $9,9(10,3-8,3)$ & $7,8(9,2-7,1)$ & $0,00^{b}$ \\
\hline Glucosa (mg/dl) ${ }^{\text {P50[RIC }]}$ & $170(207-146)$ & $119(164-90)$ & $0,00^{a}$ \\
\hline Colesterol Total (mg/dl)x(DS) & $186,67(57,12)$ & $177,67(32,14)$ & $0,33^{\mathrm{a}}$ \\
\hline $\operatorname{LDLc}(\mathrm{mg} / \mathrm{dl})^{\mathrm{x}(\mathrm{DS})}$ & $108,23(33,85)$ & $99,82(27,12)$ & $0,20^{\mathrm{b}}$ \\
\hline $\mathrm{HDLc}(\mathrm{mg} / \mathrm{dl})^{\mathrm{P} 50[\mathrm{RICl}}$ & $41(49-36)$ & $44(49-40)$ & $0,47^{a}$ \\
\hline Triglicéridos (mg/dl) (P50[RIC] $^{-1}$ & $186(219-126)$ & $165(198-131)$ & $0,93^{b}$ \\
\hline Peso $(k g)^{x(D S)}$ & $70,36(11,52)$ & $70,58(12,43)$ & $0,64^{b}$ \\
\hline IMC $\left(\mathrm{kg} / \mathrm{m}^{2}\right)^{\mathrm{x}(\mathrm{DS})}$ & $27,97(3,81)$ & $27,31(3,87)$ & $0,60^{\mathrm{b}}$ \\
\hline \multicolumn{4}{|c|}{ A los 12 meses } \\
\hline $\mathrm{HbA1C}(\%) P 50^{[R I C]}$ & $9,7(11,2-8,6)$ & $8,3(8,8-7,1)$ & $0,00^{a}$ \\
\hline Glucosa $(\mathrm{mg} / \mathrm{dl})^{\mathrm{P50}[\mathrm{RIC}]}$ & $175(223-128)$ & $130(173-102)$ & $0,00^{\mathrm{a}}$ \\
\hline Colesterol Total (mg/dl) ${ }^{\text {(DS) }}$ & $169,67(51,59)$ & $178,16(28,54)$ & $0,36^{b}$ \\
\hline $\operatorname{LDLc}(\mathrm{mg} / \mathrm{dl})^{\mathrm{x}(\mathrm{DS})}$ & $97,17(34,82)$ & $97,84(22,50)$ & $0,85^{b}$ \\
\hline $\mathrm{HDLc}(\mathrm{mg} / \mathrm{dl})^{\mathrm{P} 50[\mathrm{RICl}}$ & $41(50-35)$ & $42(48-39)$ & $0,68^{a}$ \\
\hline Triglicéridos (mg/dl) ${ }^{\text {P50[RIC] }}$ & $148(207-105)$ & $161(189-119)$ & $0,96^{\mathrm{a}}$ \\
\hline Peso $(\mathrm{kg})^{\mathrm{x}(\mathrm{DS})}$ & $71,40(11.37)$ & $71,12(12,25)$ & $0,78^{b}$ \\
\hline $\operatorname{IMC}\left(\mathrm{kg} / \mathrm{m}^{2}\right)^{\mathrm{x}(\mathrm{DS})}$ & $28,37(3,50)$ & $27,52(3,87)$ & $0,44^{b}$ \\
\hline $\begin{array}{l}\text { PED: Programa de educación diabetológica } \\
\text { IMC: índice de masa corporal; HbA1C: Hen } \\
\text { ata densidad. } \\
\text { a. variable con distribución no normal. Me } \\
\text { (Mann-Whitney), b. variable con distribuci } \\
\text { rianzas. }\end{array}$ & $\begin{array}{l}\text { nediana, RIC: rango inter } \\
\text { na glicosilada A1c; LDLC: } \\
\text { RIC, valor p con Prueba } \\
\text { nal. Media y DS, valor } p\end{array}$ & $\begin{array}{l}\text { tílico; x: promedio, DS: desviaci } \\
\text { esterol de baja densidad, HDLc } \\
\text { uma de rangos de Wilcoxon de } \\
\text { r con T student según homoge }\end{array}$ & $\begin{array}{l}\text { n estándar } \\
\text { colesterol } \\
\text { os muestr }\end{array}$ \\
\hline
\end{tabular}

Cuadro 3. Intensidad de la variación $(\Delta)$ en ambos grupos en comparación al nivel basal

\begin{tabular}{|c|c|c|c|c|c|c|}
\hline Características & $\begin{array}{c}\text { Control }(n=45) \\
\Delta 6 m\end{array}$ & $\begin{array}{c}\text { PED }(n=45) \\
\triangle 6 m\end{array}$ & Valor $p$ & $\begin{array}{c}\text { Control }(n=45) \\
\Delta 12 m\end{array}$ & $\begin{array}{c}\text { PED }(n=45) \\
\triangle 12 m\end{array}$ & Valor $p$ \\
\hline Peso ${ }^{\mathrm{p} 50[\mathrm{R} I \mathrm{C}]}$ & $-0,62(1,6 ;-1,7)$ & $-1,2(0,2 ;-2,8)$ & $0,01^{a}$ & $0,4(2 ;-1,7)$ & $-0,6(0,9 ;-2)$ & $0,13^{a}$ \\
\hline IMC ${ }^{\mathrm{p} 50[\mathrm{RIC}]}$ & $-0,23(0,66 ;-0,68)$ & $-0,43(0,08 ;-1,05)$ & $0,02^{a}$ & $-0,17(0,78 ;-0,68)$ & $-0,22(0,39 ;-0,76)$ & $0,14^{\mathrm{a}}$ \\
\hline $\mathrm{HbA} 1 \mathrm{C}$ p50[RIC] & $0,7(0,9 ;-0,6)$ & $-1,7(-0,2 ;-2,4)$ & $0,00^{\mathrm{a}}$ & $0,5(1,5 ;-0,8)$ & $-1,2(-0,5 ;-2)$ & $0,00^{\mathrm{a}}$ \\
\hline Colesterol Total ${ }^{\mathrm{p} 50[\mathrm{R} \mid \mathrm{C}]}$ & $-3(18 ;-41)$ & $-6(24 ;-38)$ & $0,98^{\mathrm{a}}$ & $-20(1 ;-40)$ & $-5(20 ;-26)$ & $0,03^{a}$ \\
\hline LDLc ${ }^{x(D S)}$ & $-5,60(37,27)$ & $-8,69(42,0)$ & $0,71^{b}$ & $-16,65(32,52)$ & $-10,66(34,74)$ & $0,71^{b}$ \\
\hline HDLc ${ }^{p 50[R I C]}$ & $-4(2,3 ;-6)$ & $5(9 ;-4)$ & $0,02^{a}$ & $-4(2,1 ;-8)$ & $3(5 ;-3)$ & $0,09^{a}$ \\
\hline
\end{tabular}

PED: Programa de educación diabetológica. p50: mediana, RIC: rango intercuartílico; x: promedio, DS: desviación estándar. 
Enero - abril de 2021 - Pág 10

La Asociación Latinoamericana de Diabetes recomienda intervenciones grupales por ser más efectivas, impartidas por un educador certificado que fomente la participación activa de los pacientes y que pueda mantenerse en forma presencial o por medios electrónicos.
La meta de $\mathrm{HbA} 1 \mathrm{c}<7 \%$ se alcanzó en el 24,4 y 22,2 \% en el grupo PED vs. 4,4 y 6,7 \% en el grupo control, a los seis y 12 meses, respectivamente. En relación a la meta de colesterol LDL (LDLC) $<100 \mathrm{mg} / \mathrm{dL}$ se encontró un aumento desde $44,4 \%$ antes de la intervención hasta 57,8 y 55,6 \% a los seis y 12 meses, respectivamente, en el grupo PED-HNERM. El 13,3\% del grupo PED alcanzó una pérdida de peso $>5 \%$ del basal en el año de seguimiento.

\section{Discusión}

El programa de educación en diabetes fue aplicado a pacientes con varios años de diagnóstico quienes se atendían en centros de atención primaria y eran referidos a la institución por mal control metabólico, esto no se condice con las recomendaciones de Asociación Latinoamericana de Diabetes (6) que sugieren ofrecer intervenciones educativas desde el momento del diagnóstico y durante el seguimiento de los pacientes.

El programa tuvo una duración de cuatro horas en total y los pacientes fueron seguidos durante año. En otros estudios la duración de la intervención y el seguimiento iban desde algunos meses hasta dos años, mostrando ser efectivos en comparación con la atención habitual en reducir la $\mathrm{HbA} 1 \mathrm{c}$ hasta $-1,4 \%$ y en mejorar la adherencia al tratamiento farmacológico y la calidad de vida (6).

Un estudio previo en el mismo hospital con 41 pacientes reporta una disminución de $\mathrm{HbA} 1 \mathrm{c}$ de 1,7 \% a los tres meses, también una disminución de HbA1c de 1,2\% a los 12 meses de seguimiento. El uso de estrategias como actividades de refuerzo presencial, telefónico o virtual durante el seguimiento no fueron parte del programa y ello podría explicar, en parte, el aumento de $+0,5 \%$ entre los 6 y 12 meses de seguimiento en el grupo PED-HNERM(13). La Asociación Latinoamericana de Diabetes recomienda intervenciones grupales por ser más efectivas, impartidas por un educador certificado que fomente la participación activa de los pacientes y que pueda mantenerse en forma presencial o por medios electrónicos (6).

Duke et al. realizan una revisión sistemática sobre educación individual con 1359 pacientes con diabetes mellitus tipo 2. La intervención fue cara a cara, mientras que los pacientes del grupo control recibieron atención habitual, tratamiento de rutina o educación grupal. Incluyeron estudios que evaluaron resultados al menos en seis meses desde el inicio; seis estudios que compararon la educación individual vs atención habitual encontraron que la intervención individual no mejoró significativamente el control glicémico (diferencia de medias ponderada (DMP) en HbA1c fue $-0,1 \%)$ durante un período de 12-18 meses. Sin embargo, parecía haber un beneficio significativo de la educación individual sobre el control glicémico en un análisis de subgrupos de tres estudios que incluyeron participantes con una HbA1c media > $8 \%$ (DMP - 0,3\%). En dos estudios que compararon la educación individual vs grupal, no hubo diferencias significativas en el control glicémico a los 12-18 meses con una diferencia de medias ponderada en $\mathrm{HbA} 1 \mathrm{c}$ de $0,03 \%$, sugiriendo un beneficio de la educación individual sobre el control glicémico en comparación con la atención habitual en aquellos con una HbA1c basal > 8 \% (15). En un centro de atención primaria del Seguro Social en México la intervención educativa DiabetIMSS promovió una disminución de HbA1c de \pm 0,69 \% en 12 meses (16); mientras que en Costa Rica el programa PINEC en 23 centros de atención primaria mostró una disminución de $\mathrm{HbA} 1 \mathrm{c}$ de \pm 0,95\% en el tiempo de seguimiento (17); ambos estudios no contaron con sendos grupos control. 
El riesgo cardiovascular en diabetes mellitus tipo 2 es directamente proporcional al grado de elevación de LDLc, por lo que representa la primera meta que debe alcanzarse.
Adaptamos las metas recomendadas por la Asociación Latinoamericana de Diabetes: IMC entre $18,5-25 \mathrm{~kg} / \mathrm{m}^{2}$ o reducciones del $10 \%$ del peso basal en personas con obesidad; la cintura menor de $94 \mathrm{~cm}$ en varones y de $90 \mathrm{~cm}$ en mujeres; la HbA1c generalmente menor de $7 \%$, pero dependiendo de las características de cada paciente puede estar dentro del rango de 6,5-8 \%; la presión arterial sistólica entre 130-140 $\mathrm{mm} \mathrm{Hg}$, y menor o igual de $80 \mathrm{~mm} \mathrm{Hg}$ la diastólica. En cuanto a la lipemia recomiendan LDLc menor de $100 \mathrm{mg} / \mathrm{dL}$ para diabetes mellitus tipo 2 sin ECV, de $70 \mathrm{mg} / \mathrm{dL}$ para los casos en prevención secundaria y menor de $55 \mathrm{mg} / \mathrm{dL}$ para casos con muy alto riesgo; el valor de los triglicéridos menor $150 \mathrm{mg} / \mathrm{dL}$ y HDLc mayor de $40 \mathrm{mg} / \mathrm{dL}$. Para la mayoría de estos parámetros no existe un umbral por debajo del cual se pueda asegurar que la persona no desarrollará complicaciones (6).

En cuanto al peso, el grupo de intervención alcanzó una pérdida ponderal de -0,6 kg al año y una pérdida de peso mayor de $5 \%$ del nivel basal en $13,3 \%$ de pacientes al año. En el estudio Look-AHEAD los pacientes con diabetes mellitus tipo 2 que fueron sometidos a una intervención intensiva perdieron en promedio $8,6 \%$ del peso en el primer año (6,2 \% durante los cuatro años), con lo cual mejoró significativamente el control de glicemia, presión arterial, HDLc y triglicéridos (18).

En relación a la meta de $\mathrm{HbA1c}$, el estudio ACCORD, en pacientes con un promedio de edad de 62 años no mostró beneficio en el grupo que alcanzó niveles de HbA1c alrededor de 6,4 \%; por el contrario, encontraron una mayor tasa de mortalidad al alcanzar esta meta (19). El estudio VADT no halla beneficio significativo en reducción global de complicaciones micro o macrovasculares con la reducción de $-1,5 \%$ de HbA1c, en pacientes de 60 años en promedio (20).

El estudio ADVANCE planteó la meta de 6,5 \% para la HbA1c e incluyó pacientes con diabetes mellitus tipo 2 de menor duración que ACCORD (8 vs 10 años), encontrando una reducción de complicaciones macro y microvasculares-(21). Y el estudio UKPDS halla una reducción de los eventos macrovasculares con el tratamiento intensivo, que sólo se manifestó 10 años después de finalizado el estudio (22), a pesar de que las diferencias de $\mathrm{HbA} 1 \mathrm{c}$ entre el grupo intensivo y el convencional se perdieron un año después de finalizado el estudio. En el presente estudio el grupo de intervención presentó un incremento importante en el número de pacientes (22,2 \%) que alcanzaron una $\mathrm{HbA} 1 \mathrm{c}<7 \%$ al año de la intervención.

En cuanto a la glicemia en ayunas se encontró una disminución de $-56 \mathrm{mg} / \mathrm{dL}$ a los 12 meses, la cual puede no traducir un adecuado control metabólico. La Asociación Latinoamericana de Diabetes recomienda el automonitoreo glicémico en los usuarios de insulina, en quienes el ajuste de la dosis debe realizarse en base a este control, pero estos equipos no estuvieron disponibles.

El riesgo cardiovascular en diabetes mellitus tipo 2 es directamente proporcional al grado de elevación de LDLc (6), por lo que representa la primera meta que debe alcanzarse. Para alcanzar las metas en el perfil lipídico se deben promover cambios en el estilo de vida y el uso de hipolipemiantes, puesto que son efectivos para reducir la incidencia de nuevos eventos cardiovascular en pacientes con diabetes mellitus tipo 2, cuando se alcanzan concentraciones de LDLc menores de $100 \mathrm{mg} /$ $\mathrm{dL}$ (6). En este estudio, la mayoría recibía estatinas antes del programa, un año después un $53,9 \%$ alcanzó metas de LDLc, un $62,2 \%$ metas de HDLc y un $40 \%$ metas 
Un programa de educación en diabetes añadido al tratamiento habitual especializado del paciente con diabetes mellitus permitió una disminución importante y sostenible de la HbA1c y la glicemia, una mejora del perfil lipídico. de triglicéridos, demostrando un efecto beneficioso sobre los lípidos, quedando un grupo significativo fuera de metas y en quienes se podría optar por otras opciones farmacológicas si están disponibles (23).

En cuanto a la intensidad de los cambios, se observó una disminución de HbA1c de $-1,2 \%$ a los 12 meses; mayor a los reportados por Steinsbekk et al., quienes comparan la educación grupal vs el tratamiento habitual y donde la intervención tuvo al menos una sesión y al menos seis meses de seguimiento. Incluyeron 2833 pacientes, la edad basal era de 60 años, IMC: $31,6 \mathrm{~kg} / \mathrm{m}^{2}$, HbA1c: $8,23 \%$, diabetes mellitus de ocho años y $82 \%$ usaban medicamentos. La HbA1c se redujo significativamente a los seis meses $(-0,44 \%), 12$ meses $(-0,46 \%)$ y dos años $(-0,87 \%)$ y los niveles de glicemia en ayunas también se redujeron significativamente a los 12 meses (-22,6 $\mathrm{mg} / \mathrm{dl}$ ), pero no a los seis meses. Según este estudio las intervenciones realizadas por un solo educador, entregadas en menos de diez meses, con más de 12 horas y entre 6 y 10 sesiones dan los mejores resultados (24).

Los resultados del presente estudio son similares a los reportados por Deakin et al., en un estudio con 1532 pacientes, quienes encuentran que los programas de educación grupal permiten una reducción de HbA1c a los 4-6 meses (-1,4\%), a los 12-14 meses. $(-0,8 \%)$ y dos años $(-1,0 \%)$; reducción de glicemia en ayunas a los 12 meses $(-21,6 \mathrm{mg} / \mathrm{dL})$; reducción del peso a los $12-14$ meses $(-1,6 \mathrm{~kg})$, concluyendo que la capacitación grupal en personas con diabetes mellitus tipo 2 es efectiva al mejorar la glicemia, HbA1c, el conocimiento de la enfermedad y reducir la presión arterial sistólica, el peso y la necesidad de antidiabéticos (25).

Estos hallazgos demuestran efectos beneficiosos, pero reconocemos las siguientes limitaciones: los sesgos propios del diseño cuasi-experimental, la falta de evaluación del nivel de conocimientos, la ausencia de evaluación del grado de adherencia, la falta de actividades de refuerzo pos-intervención, la no participación de otros profesionales de la salud, la no evaluación de otros parámetros como la cintura y presión arterial y la falta de monitoreo glicémico. Para aumentar la potencia de estos hallazgos y su extrapolación se deben realizar más estudios con un mayor número de pacientes y un mayor periodo de seguimiento.

\section{Conclusiones}

Un programa de educación en diabetes añadido al tratamiento habitual especializado del paciente con diabetes mellitus permitió una disminución importante y sostenible de la HbA1c y la glicemia, una mejora del perfil lipídico; además, de una discreta pérdida de peso al año de seguimiento.

\section{Bibliografía}

1. IDF Diabetes Atlas Committee. Global and regional diabetes prevalence estimates for 2019 and projections for 2030 and 2045: Results from the International Diabetes Federation Diabetes Atlas, 9th edition. Diabetes Res Clin Pract. 2019;157:107843. DOI: 10.1016/j.diabres.2019.107843

2. Seclen S, Rosas M, Arias A, Medina C. Prevalence of diabetes and impaired fasting glucose in Peru: report from PERUDIAB, a national urban population-based longitudinal study. BMJ Open Diabetes Research and Care 2015;3: e000110. DOI: 10.1136/bmjdrc-2015-000110 
3. Ramos W, López T, Revilla L, More L, Huamaní M, Pozo M. Resultados de la vigilancia epidemiológica de diabetes mellitus en hospitales notificantes del Perú, 2012. Rev Per Med Exp Salud Pública. 2014;31(1): 09-15.

4. Casanova M, Bayarre H, Navarro D, Sanabria G, Trasancos M. Educación diabetológica, adherencia terapéutica y proveedores de salud. Rev Cub Salud Pública. 2015;41(4):677-680.

5. Coronel M, Córdova C, Delgado M, Sánchez W. Educación terapéutica sobre diabetes mellitus: pilar esencial del tratamiento. Rev Cient Mundo Invest Conoc. 2019; 3(1): 38-57.

6. Asociación Latinoamericana de Diabetes. Guías ALAD sobre el diagnóstico, control y tratamiento de la diabetes mellitus tipo 2 con medicina basada en evidencia. Rev ALAD. 2019. Disponible en: http://www.revistaalad.com/ guias/5600AX191_guias alad_2019.pdf

7. Deakin TA, Cade JE, Williams R, Greenwood DC. Structured patient education: the diabetes X-PERT Programme makes a difference. Diab Med. 2006; 3(9):944-954. DOI: 10.1111/j.1464-5491.2006.01906.x

8. Khunti K, Gray LJ, Skinner T, Carey ME, Realf K, Dallosso H, et al. Effectiveness of a diabetes education and self management programme (DESMOND) for people with newly diagnosed type 2 diabetes mellitus: three year follow-up of a cluster randomised controlled trial in primary care. BMJ 2012;344:e2333. DOI: https://doi.org/10.1136/bmi.e2333

9. Fernández S. Determinación del tamaño muestral. Unidad de Epidemiología Clínica y Bioestadística. Complexo Hospitalario Universitario de A Coruña. Cadernos de Atención Primaria. 1996; 3,138-14. Actualizada el 01/12/2010. Disponible en: http://www.fsterra.com/mbe/investiga/ 9muestras/9muestras2.asp

10. American Association of Diabetes Educators. AADE Guidelines for the Practice of Diabetes Self-Management Education and Training (DSME/T). Diab Educ. 2009;35(3_suppl):85S-107S. DOI:10.1177/0145721709352436

11. American Association of Diabetes Educators. AADE $7^{\circ}$ Self-Care behaviors american association of diabetes educators position statement. 2014. Disponible en: https://www.diabeteseducator.org/docs/default-source/ legacy-docs/resources/pdf/publications/aade7 position statement final.pdf?sfvrsn=4

12. American Diabetes Association. Standards of Medical Care in Diabetes. Diab Care. 2014; 37 (Suppl 1),S14-80. DOI: https://doi.org/10.2337/dc14-S014

13. Paz-Ibarra J, Benites R. Efecto de un programa de educación diabetológica 
en pacientes adultos de la consulta ambulatoria en un hospital nacional peruano. An Fac Med. 2020;81(2):161-6. DOI: https://doi.org/10.15381/ anales.v81i2.17673

14. Hernández S, García C, Mehta R, Aguilar C, Kershenobich D. Innovative models for the empowerment of patients with type 2 Diabetes: The CAIPaDi Program. Recent Patents Endocrine, Metabolic \& Immune Drug Discovery, 2014; 8(3), 202-209.

15. Duke SA, Colagiuri S, Colagiuri R. Individual patient education for people with type 2 diabetes mellitus. Cochrane Database System Reviews. 2009; 1:CD005268

16. Mendoza M, Velasco J, Nieva R, Andrade H, Rodríguez C, Palou E. Impacto de un programa institucional educativo en el control del paciente diabético. Rev Med Inst Mex Seguro Soc. 2013;51(3):254-9

17. Roselló M, Guzmán S, Aráuz A, Arias D, Schmidt M, Vargas M. Efecto de la metodología de educación terapéutica, mediante la evaluación de los cambios bioquímicos, antropométricos y dietéticos en las personas con diabetes mellitus tipo 2. Rev ALAD. 2018;8(3):99-109. DOI: 10.24875/ ALAD.M18000006

18. Wing R, Bahnson J, Bray G, Clark J, Coday M, Egan C for the Look AHEAD Research Group. Long-term effects of a lifestyle intervention on weight and cardiovascular risk factors in individuals with type 2 diabetes mellitus: four-year results of the Look AHEAD trial. Arch Intern Med. 2010 Sep 27:170(17):1566-75. doi: 10.1001/archinternmed.2010.334

19. Gerstein HC, Miller ME, Byington RP, Goff D, Bigger T, Buse J, et al for the Action to Control Cardiovascular Risk in Diabetes Study Group. Effects of intensive glucose lowering in type 2 diabetes. N Engl J Med. 2008; 358(24):2545-59. DOI: 10.1056/NEJMoa0802743

20. Duckworth W, Abraira A, Moritz T, Reda D, Emanuele N, Reaven P, et al., for the VADT Investigators. Glucose control and vascular complications in veterans with type 2 diabetes. N Engl J Med. 2009;360(2):129-39. DOl: 10.1056/NEJMoa0808431.

21. Patel A, MacMahon S, Chalmers J, Neal B, Billot L, Woodward M, et al for the ADVANCE Collaborative Group. Intensive blood glucose control and vascular outcomes in patients with type 2 diabetes. N Engl J Med. 2008; 12;358(24):2560-72. DOI: 10.1056/NEJMoa0802987

22. Holman R, Paul S, Bethel M, Matthews D, Neil H. 10-year follow-up of intensive glucose control in type 2 diabetes. N Engl J Med. 2008 Oct;359(15):1577-1589. DOI: 10.1056/nejmoa0806470 
23. Castillo Y, Aguilar C, Mendivil C, Rodríguez M, Lyra R. Consenso del Grupo de Tareas de la Asociación Latinoamericana de Diabetes (ALAD) sobre el diagnóstico y manejo de la dislipidemia diabética. Rev ALAD. 2018; 8(3):118-140.

24. Steinsbekk, A., Rygg, L., Lisulo, M. Group based diabetes self-management education compared to routine treatment for people with type 2 diabetes mellitus. A systematic review with meta-analysis. BMC Health Serv Res. 2013; 12, 213. https://doi.org/10.1186/1472-6963-12-213

25. Deakin T, McShane C, Cade J, Williams R. Group based training for self-management strategies in people with type 2 diabetes mellitus. Cochrane Database System Reviews. 2005. 2:CD003417 